\title{
Microtension control for a yarn winding system with an IMC PID controller
}

\author{
Qing Wang ${ }^{1,}$, Anqing $\mathrm{Li}^{2}$, Yuanyuan $\mathrm{Li}^{3}$, Jixin $\mathrm{Liu}^{3}$, Hui Shen ${ }^{1}$, and Guoyong $\mathrm{Li}^{4}$ \\ ${ }^{1}$ School of Electromechanic Engineering, Qingdao University, Qingdao, Shandong, PR China \\ ${ }^{2}$ School of Mechanical \& Automotive Engineering, Qilu University of Technology, Jinan, Shandong, PR China \\ 3 Mechanical and Electrical Engineering College, Qingdao Huanghai University, Qingdao, Shandong, PR China \\ ${ }^{4}$ Shandong food and drug center for certification and evaluation, Jinan, Shandong, PR China
}

Received: 24 October 2018 / Accepted: 2 September 2019

\begin{abstract}
In this paper, feedforward compensation and an internal model control (IMC) PID tuning method to maintain the yarn tension within a micro-boundary range are proposed. The proposed method can be used to improve the quality of products in textile industry. We first develop a mathematical model of the AC servo motor and yarn tension system. Based on the results of the mathematical model, an IMC PID controller is designed to control the microtension of the yarn. The proposed IMC-PID controller can be directly calculated from the time constant and time delay. Feedforward control is used to compensate for the linear velocity of the winding roller. To reduce the lateral vibrations of the yarn, we designed an active roller to nip the moving yarn. The active roller compensates for the variation in the diameter of the unwinding roller. The proposed method effectively improves the dynamics performance and the robustness of the system, and is appropriate for industrial application. Experimental instruments, including a tension sensor, an AC servo motor and a motion controller, equipped with a computer, are used to test the proposed method. The simulation and experimental results show the effectiveness of the proposed controller for the yarn microtension control system.
\end{abstract}

Keywords: IMC PID controller / feedforward compensation / microtension control / active roller / $\mathrm{AC}$ servo motor

\section{Introduction}

Yarn tension is crucial parameter in the winding process. The unwinder-winder system can be considered an axially moving string system, and the lateral vibration of the moving string causes yarn tension to fluctuate continuously. In the process of production of yarn, the breakage of the product is crucial for all technological processes associated with the processing of threads [1]. Breakage in spinning is a most important factor, causing a decrease in the productivity as well as an increase in loss of raw material. Yarn break detection at the right time is one of the important levels of the yarn production and weaving stages. The spinning process can run continuously only when there are no breaks in the output yarn [2-5]. However, yarns do break, the main reason for breakage is that the yarn tension and fluctuations exceed its allowable value [6]. In the yarn winding process, maintaining the yarn tension within a bounded range is an important technique

\footnotetext{
* email: wangqing17@qdu.edu.cn
}

to maintain the fabric properties of the textile. To achieve high quality textiles in the winding process, the winding machine must be equipped with a tension controller $[7,8]$. Specifically, active string tension control in the winding process requires using the string tension as a feedback signal, and then adjusting the tension using a tension controller [9]. Yarn tension is a function of the winding velocity, and the tension is adjusted by changing the speed of the yarn. According to Maryam Naebe [10], we know that a smaller yarn tension result in, a better predictor of the fabric's Wool ComfortMeter value used in the yarn winding process. In addition, the tension of the string affects the nip dimensions and string diameter as the yarn stretches $[11,12]$. These considerations demonstrate the importance of keeping the yarn tension within a microboundary range. It is therefore important to design a novel method of tuning the control structure of the microtension control system.

The yarn tension control system finds applications in many textile processes including bobbin winding, warp sizing, dyeing, warp preparation for weaving and warp knitting. Many methods have been developed in the past to 


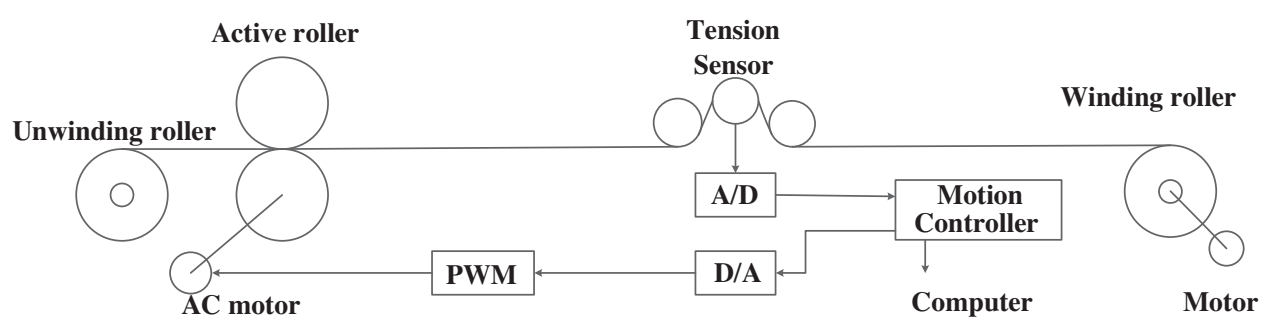

Fig. 1. Structure of the tension control system.

control the winding tension of the yarn and web. Lin [13] proposed an observer which was able to estimate web tension precisely. Knittel et al. [14] presented multivariable robust control with $2 \mathrm{DOF}$ applied to winding systems. Wang et al. [7] applied neural networks to the system. In this paper, they study and create a tension control system. Vivek [15] designed a device that ensured uniform tension in the yarn while the yarn was being twisted. Gassmann et al. [16] proposed an improved alternative based on methods that provide a systematic framework. A pendulum dancer was applied in the unwinding section of a processing line. In these experiments, the transient response of the tension control system had a prolonged regulation process and noticeable vibrations. However, the existing research on yarn tension modeling has neglected the time delay of the winding system, and they have been mainly focused on large tension levels.

It is common practice in the industrial yarn unwinderwinder processes to use a PID controller. The ratio of applications of PID control, conventional advanced control, and model predictive control is approximately 100:10:1 [17]. However, selecting the parameters of the PID controller is difficult [18]. As a result, tuning the PID controller is necessary. The direct synthesis methods [19] and IMC-PID tuning methods [14] are two typical tuning methods. The IMC-PID tuning rule, which also considers time delay, has only one adjustable parameter. Shamsuzzoha et al. [20] proposed a simple method for PID controller tuning using closed-loop experiments. Mozhdehi et al. [21] proposed a method to tune PIDs for non-linear systems.

In the above studies, one aspect of the tuning method was choosing the maximum sensitivity, and another aspect was calculating the adjustable parameters by contrasting the control performances in simulation studies. The researchers found that it was necessary to design more controllers with more tuning parameters.

In this paper, we proposed a new IMC PID controller for yarn winding system. The adjusting parameter $\lambda$ was directly calculated by the time constant and time delay of the processes. The main purpose of our work is to maintain the yarn tension at a micro level, and the method of tuning the coefficient of the controller is the main problem to resolve. Our procedure was organized in the following steps. First, the mathematical model of the microtension control system was established and simplified to a first order integration process with a time delay. The mathematical model was established by the yarn velocities and nipping action of active rollers. Then, the IMC tuning rule was designed to tune the parameters of the PID controller for the yarn tension control system. Finally, using system identification and curve fitting, we obtained the time constant of the velocity loop and tension sensor, and the simulation and experiment of the winding-unwinding system were carried out.

\section{System model}

\subsection{Microtension control system}

Figure 1 shows the structure of the microtension control system. Given the properties of roll-to-roll web winding systems and the goal of maintain the yarn tension within a micro-boundary range, we proposed tests on the active roller, of the yarn winding system. In the microtension control system, one AC servo motor is attached to the active roller, and the other AC servo motor is attached to the winding roller. The radius of the active roller is constant and couples the linear velocity to the angular velocity of the roller, which makes the active roller easier to control than the unwinding roller. The yarn is clamped and driven forward by the active roller, while the active roller and the winding roller are driven by $\mathrm{AC}$ servo motors. Because the yarn is nipped by the active roller, we can ignore vibration from the unwinding roller and the yarn tension between the unwinding roller and the active roller. We define the distance between the active roller and the winding roller as $l_{0}$, the linear velocity of the active roller as $v_{1}$, and the linear velocity of the winding roller as $v_{0}$. Because the linear velocities of the active roller and winding roller is different, the yarn is stretched, which generates tension. Because the yarn tension in this study is kept within a micro-boundary range, the addition of the yarn to a winding roller can be treated as a linear analysis using elastic strain theory within the model, which conforms to Hooke's law. It is represented by

$$
\sigma=E \varepsilon=E \frac{\int\left(v_{1}-v_{0}\right) d t}{l_{0}}
$$

where $E$ is the Young's modulus of yarn, $\varepsilon$ is the yarn strain, and $\sigma$ is the yarn tensile stress. And $\varepsilon$ can be calculated by $\varepsilon=\frac{\Delta l}{l_{0}}=\frac{\int\left(v_{1}-v_{0}\right) d t}{l_{0}}$.

The tension $F$ can be described by [22]

$$
F=E A \frac{\int\left(v_{1}-v_{0}\right) d t}{l_{0}}=\frac{\pi d_{0}^{2} E}{4 l_{0}} \int\left(v_{1}-v_{0}\right) d t
$$




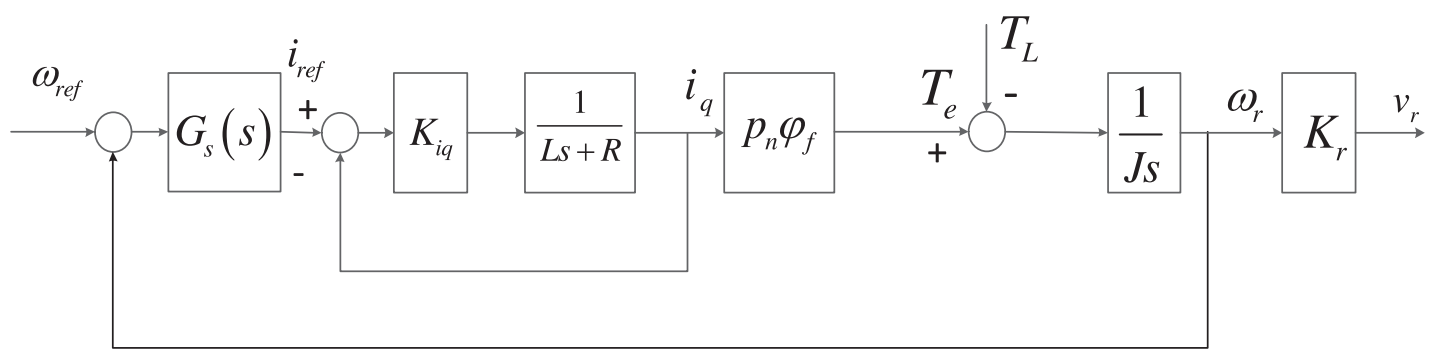

Fig. 2. AC servo motor equivalent simplify model.

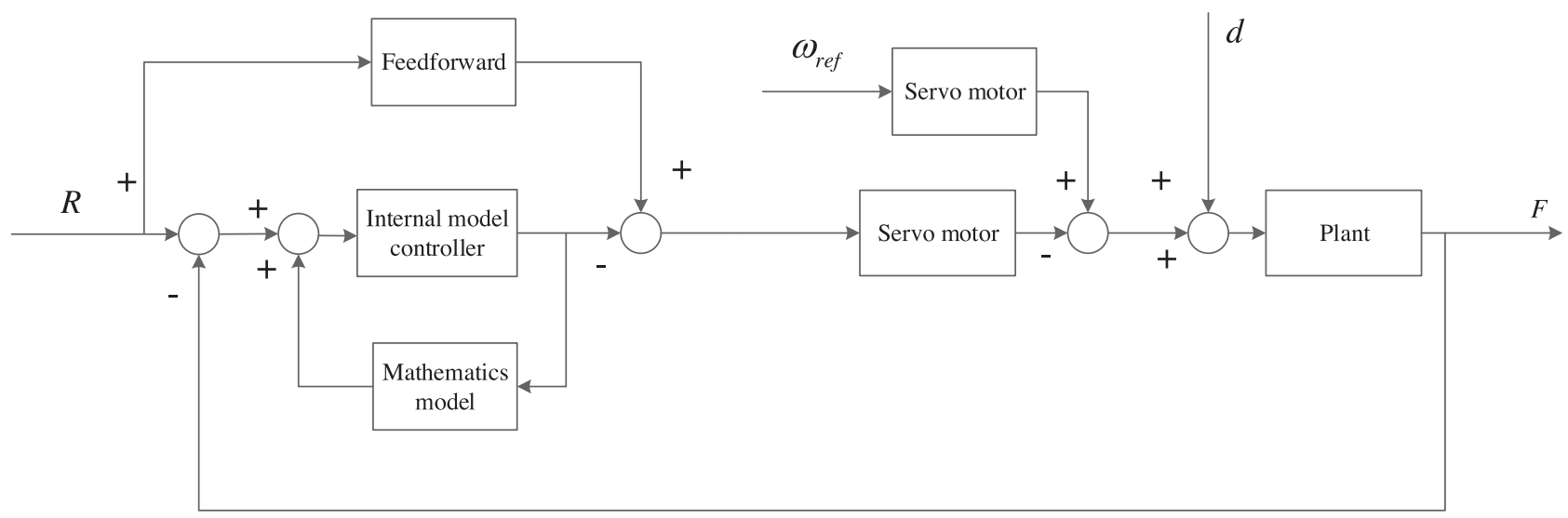

Fig. 3. Feedforward and IMC control system.

where $A$ is the cross-sectional area of the yarn section. Then, we can obtain:

$$
\frac{d F}{d t}=\frac{\pi d_{0}^{2} E}{4 l_{0}}\left(v_{1}-v_{0}\right)
$$

The transfer function of the yarn tension system is as follows:

$$
F(s)=\frac{\pi d_{0}^{2} E}{4 l_{0}} \frac{1}{s}\left[V_{1}(s)-V_{0}(s)\right]
$$

Based on equations (3) and (4), the yarn tension clearly depends on the difference of $v_{1}$ and $v_{0}$.

\subsection{AC servo motor}

A permanent magnet synchronous motor (PMSM) servo system $\left(L_{d}=L_{q}=L\right)$ was used to apply an $i_{d}=0$ current control method.

The dynamic equilibrium equations of the PMSM servo motor are as follows [7]:

$$
\left\{\begin{array}{l}
\frac{d}{d t} i_{d}=\frac{1}{L_{d}} u_{d}-\frac{R}{L_{d}} i_{d}+\frac{L_{q}}{L_{d}} p_{n} \omega i_{q} \\
\frac{d}{d t} i_{q}=\frac{1}{L_{q}} u_{q}-\frac{R}{L_{q}} i_{q}-\frac{L_{d}}{L_{q}} p_{n} \omega i_{d}-\frac{\phi_{f} p_{n} \omega}{L_{q}} \\
J \frac{d \omega_{r}}{d t}=T_{e}-B \omega_{r}-T_{L}
\end{array}\right.
$$

In equation (5), $L_{d}$ and $L_{q}$ are the $d-q$ axis stator inductance, $J$ is the rotational inertia of the rotor, $T_{L}$ is the load moment, $T_{e}$ is the output torque, $B$ is the viscous friction coefficient, $\omega_{r}$ is the angular velocity of rotor, $\omega=p_{n} \omega_{r}$ is the electric angular velocity of the rotor, and $p_{n}$ is the number of pole pairs. $K_{r}$ is the reducer coefficient, and $T_{v}$ is the time constant of the velocity servo system.

The mathematical model of the AC servo motor can be obtained with the vector control and current coupling method. The state equation is as follows [7]:

$$
\frac{d}{d t}\left(\begin{array}{c}
i_{q} \\
\omega_{r}
\end{array}\right)=\left(\begin{array}{cc}
-\frac{R}{L} & -\frac{p_{n} \varphi_{f}}{L} \\
\frac{p_{n} \varphi_{f}}{J} & 0
\end{array}\right)\left(\begin{array}{c}
i_{q} \\
\omega_{r}
\end{array}\right)+\left(\begin{array}{c}
\frac{u_{d}}{L} \\
-\frac{T_{L}}{J}
\end{array}\right)
$$

The PMSM velocity servo system using the vector control method consists of a current loop and velocity loop [23], which is shown in Figure 2.

In consideration of the engineering design, we can simplify the velocity servo system to a first order equation.

$$
G_{v}(s)=\frac{K_{v}}{T_{v} s+1} .
$$

\section{Design of the IMC PID controller}

Figure 3 show the feed forward and IMC control system. Based on the above discussion, we know that the winding 
process is an integral operation in the roll-to-roll system and that the winding process is also related to the difference between the linear velocities of the rollers. We also know that yarn tension is determined by the winding velocity. We therefore use a tension sensor to measure the yarn tension as a feedback signal and then adjust the tension by changing the speed of the yarn.

A feedforward compensation loop is used in the yarn tension control system. Feedforward compensation is a method that measures disturbances directly rather than basing compensations on errors, and the method provides approximate corrections to disturbances before they cause errors, therefore avoiding the development of disturbances over time. The linear velocity of the winding roller is constant and observable [13]. Therefore, feedforward control can be used to adjust the linear velocity of the winding roller. Knowing the linear velocity of the winding roller, we can adjust the linear velocity of the active roller in advance. The system includes a tension sensor and moving yarn. Any disturbance due to the gravity of the yarn or friction between the yarn and the tension sensor will be detected as a load between the rollers and the tension sensor. $R$ is the reference input tension, $\omega_{\text {ref }}$ is the reference angular velocity of the AC servo motor, $F$ is the controlled system output tension, and $d$ is the disturbance.

At present, typical methods to measure the yarn tension in the winding process involve the use of a resistance strain sensor or a capacitive sensor. In this paper, we chose the three-point yarn tension sensor to measure yarn tension. The tension sensor transfer function is described as follows:

$$
S(s)=\frac{1}{T_{s} s+1} e^{-\theta s} .
$$

The microtension control system transfer function is:

$$
P(s)=\frac{\pi d_{0}^{2} E K_{v}}{4 l_{0} s\left(T_{v} s+1\right)\left(T_{s} s+1\right)} e^{-\theta s} .
$$

$P(s)$ is the yarn winding process, which contains the $\mathrm{AC}$ servo motor and tension sensor transfer function with time delay $e^{-\theta s}$. The tension control system can be described by a second order integration with time delays.

The internal model control has a natural robustness, and it can overcome the drawbacks of time delays inherent in conventional PID controllers.

According to the design procedure for the IMC system, the model is factorized as [24]

$$
M(s)=M_{+}(s) M_{-}(s) .
$$

$M(s)$ is the process model, which is used as the internal model, and $M_{+}(s)$ and $M_{-}(s)$ are the portions of the model that are inverted and not inverted, respectively.

$$
M(s)=P(s)=\frac{\pi d_{0}^{2} E K_{v}}{4 l_{0} s\left(T_{v} s+1\right)\left(T_{s} s+1\right)} e^{-\theta s} .
$$

Define $K=\frac{\pi d_{0}^{2} E K_{v}}{4 l_{0}}$ and $T=T_{v}+T_{s}$.
According to the dominant pole theory [25], and considering the stability of the system, we can simplify $M(s)$ to a first order integration with time delays:

$$
M(s)=\frac{K}{s(T s+1)} e^{-\theta s}
$$

$f(s)$ is a low-pass filter, which we define as follows:

$$
f(s)=\frac{1+\alpha s}{(1+\lambda s)^{2}}(\alpha>0) \text {. }
$$

$Q(s)$ therefore can be designed as:

$$
Q(s)=f(s) / M_{-}(s)=\frac{s(T s+1)(\alpha s+1)}{K(\lambda s+1)^{2}} .
$$

The time delay term is approximated by the first order Padé expansion $e^{-\theta s} \approx(1-0.5 \theta s) /(1+0.5 \theta s)$. The integrating process can be approximated by a first order process [19]:

$$
\frac{K}{s} \approx \frac{\eta K}{\eta s+1}
$$

The controller can then be written as follows:

$$
C(s) \approx \frac{(\alpha s+1)(\eta s+1)(T s+1) / \eta K}{(\lambda s+1)^{2}-(\alpha s+1)(1-0.5 \theta s) /(1+0.5 \theta s)}
$$

After simplification, we can obtain the coefficient of $\alpha$ :

$$
\alpha=\frac{(2 \lambda+\theta) \eta^{2}+0.5 \theta \lambda^{2}-\left(\lambda^{2}+\theta \lambda\right) \eta}{\eta^{2}+0.5 \theta \eta} .
$$

The PID controller can be written:

$$
C(s)=K_{c}\left(1+\frac{\alpha T}{\alpha+T} s+\frac{1}{\alpha+T} \frac{1}{s}\right) \frac{1+0.5 \theta s}{1+\beta s}
$$

where $K_{c}=\frac{(\alpha+T)}{K \eta(2 \lambda+\theta-\alpha)}$ and $\beta=\frac{0.5 \theta \lambda^{2}}{\eta(2 \lambda+\theta-\alpha)}$.

The PID controller is often implemented as follows [24]:

$$
u(t)=K_{P}(\zeta r-y)+\xi K_{I} \int e(t) d t+K_{D} \frac{d e(t)}{d t}
$$

where $e(t)=R-F$ is the error signal between the reference input tension $R$ and the controlled output tension $F$, and $K_{P}, K_{I}$, and $K_{D}$ are constant $P, I, D$ control gains, respectively. $\zeta$ is the set-point weighting coefficient. Because of the time delay of the winding system, the integration of the PID controller will accumulate, eventually causing large overshoot of the system.

To solve this problem, we considered separating the integration from the control algorithm, and defined the weighting coefficient of the integration term as $\xi=1$ or 0 .

The main objective for the microtension control system is to reduce large overshoot and disturbance rejection. The yarn tension control loop relationship for input changes is 
Table 1. IMC-PID controller tuning rules.

\begin{tabular}{lllllll}
\hline Process & $\lambda$ & $\alpha$ & $K_{P}$ & $K_{I}$ & $K_{D}$ & Compensator \\
\hline$\frac{K}{s(T s+1)} e^{-\theta s}$ & $T+\sqrt{\theta T+T^{2}}$ & $\frac{(2 \lambda+\theta) \eta^{2}+0.5 \theta \lambda^{2}-\left(\lambda^{2}+\theta \lambda\right)}{\eta^{2}+0.5 \theta \eta}$ & $\frac{(1+\lambda)(\alpha+T)}{K \eta(2 \lambda+\theta-\alpha)}$ & $\frac{(1+2 \lambda)}{K \eta(2 \lambda+\theta-\alpha)}$ & $\frac{\alpha T}{K \eta(2 \lambda+\theta-\alpha)}$ & $\frac{1+0.5 \theta s}{1+0.5 \theta \lambda^{2} /(\eta(2 \lambda+\theta-\alpha)) s}$ \\
\hline
\end{tabular}

given by [20]

$$
\frac{F(s)}{R(s)}=\frac{C(s) G_{v}(s) G(s)}{1+C(s) G_{v}(s) G(s)} .
$$

The closed loop relationship for the disturbance is given by [25]

$$
\frac{F(s)}{D(s)}=\frac{F(s)}{R(s)} \frac{1}{C(s) G_{v}(s)} .
$$

From equation (21), we know that the coefficient of $\frac{1}{C(s) G_{v}(s)}$ influence the disturbance rejection directly. The optimal value of the coefficient is determined by two tradeoffs: first, between improving the dynamic performance and enhancing disturbance rejection, and second, between stability and robustness. Considering what has been discussed above, proportional-integral weighting is considered. Based on extensive simulations conducted on the yarn tension processes the weighting coefficients are given as follows:

$$
\begin{gathered}
K_{P}=(1+\lambda) \frac{\alpha+T}{K \eta(2 \lambda+\theta-\alpha)} \\
K_{I}=(1+2 \lambda) \frac{1}{K \eta(2 \lambda+\theta-\alpha)} \\
K_{D}=\frac{\alpha T}{K \eta(2 \lambda+\theta-\alpha)} .
\end{gathered}
$$

From equations (18), (22), (23) and (24), we can obtain the value of $K_{P}, K_{I}$, and $K_{D}$ (Tab. 1 ).

\section{Simulation and experiment}

The motion controller sends a step signal to the AC servo motor, and the computer collect the encoder output. According to the theory of system identification and curve fitting, we can obtain the time constant of the velocity loop (i.e. $T_{v}$ ) with the MATLAB system Identification Toolbox. Given the time delay of the AC servo motor and tension sensor, we tune the time and response axis to obtain the time constant. The result is shown in Figure 4. Using the same method, we also obtain the time constant of the tension sensor (i.e., $T_{s}$ ).

According to Figure 4 , we can calculate that $T_{v}=0.02 \mathrm{~s}$, and using the same method, we can also obtain the time constant of the tension sensor, $T_{s}=0.067 \mathrm{~s}$. From equation (12), we find that $T=0.087 \mathrm{~s}$. We choose $\eta=1000$ and $\zeta=0.4$ as the weight coefficient of the IMC PID controller. According to the experimental result, the time delay of the microtension control system is $\theta=0.1 \mathrm{~s}$.

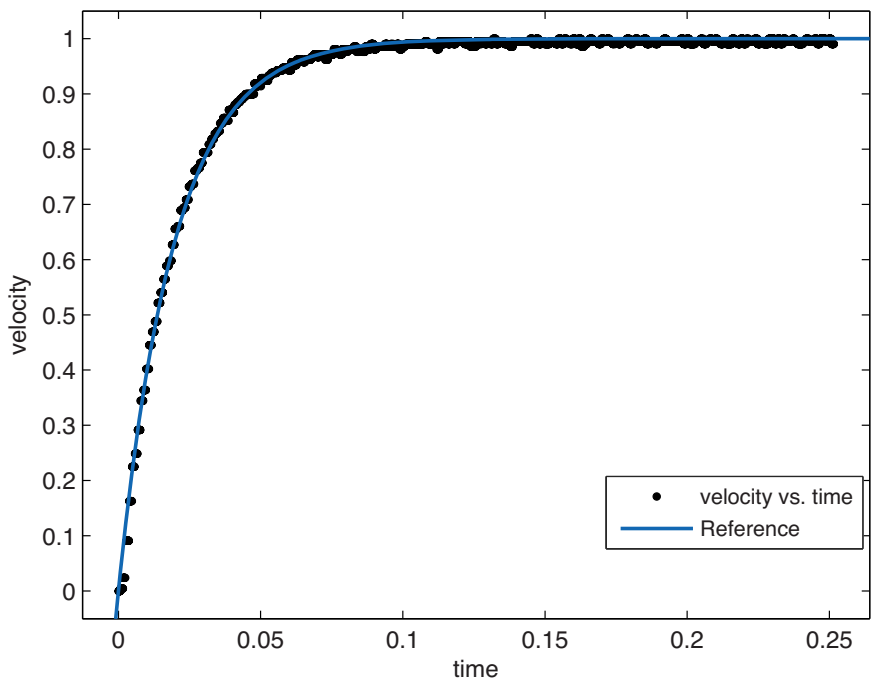

Fig. 4. Identification curve of the AC servo motor time constant.

The yarn parameters are given as $E=66.7 \mathrm{MPa}$, $l_{0}=0.23 \mathrm{~m}, r=0.5 \mathrm{~mm}$, and $\rho=0.6 \mathrm{~g} / \mathrm{m}$.

From equation (12), we can obtain $K=\frac{\pi d_{0}^{2} E K_{v}}{4 l_{0}}=61.76$.

We can then confirm the microtension control system transfer function

$$
M(s)=P(s)=\frac{61.76}{s(0.087 s+1)} e^{-0.1 s} .
$$

The parameters of the IMC PID controller are obtained below.

$\lambda=T+\sqrt{\theta T+T^{2}}=0.21455$ and $\alpha=0.529$.

Based on the above calculations and according to equation (19), (23) and (24), the parameters of the IMC PID controller assume the following values: proportional coefficient $K_{P}=0.121$, integral coefficient $K_{I}=0.231$ and differential coefficient $K_{D}=0.0075$. To demonstrate the effectiveness of the proposed IMC PID controller, the simulation results were compared with the method proposed by SIMC [20], Chen and Seborg [26] and Shamsuzzoha and Lee [27]. To provide a fair comparison, the maximum sensitivity, defined as $M s=\max |1 /[1+P(j w) C(j w)]|$, is used. In this paper, we choose $M s=2.26$. The results of the simulation were shown in Figure 5.

To evaluate the robustness of the process, a $10 \%$ change in system delay and the steady state gain are assumed, such as $P(s)=\frac{67.936}{s(0.087 s+1)} e^{-0.11 s}$. The results of the simulation are shown in Figure 6, and the performance indices are providing in Table 2.

From the simulation results we can clearly see that improved performance is obtained with the proposed method. 


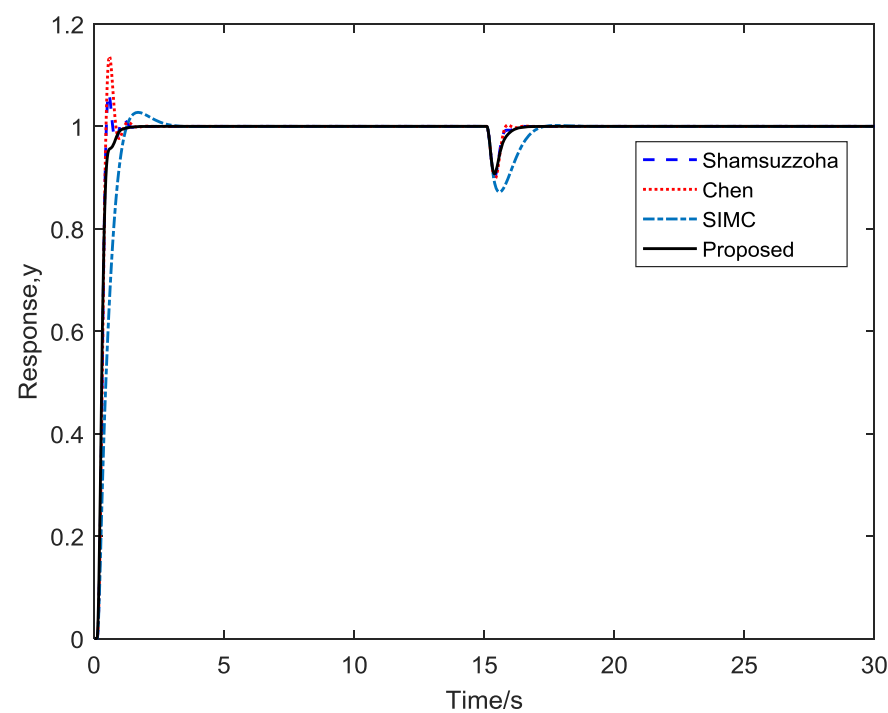

Fig. 5. Response for yarn tension control system.

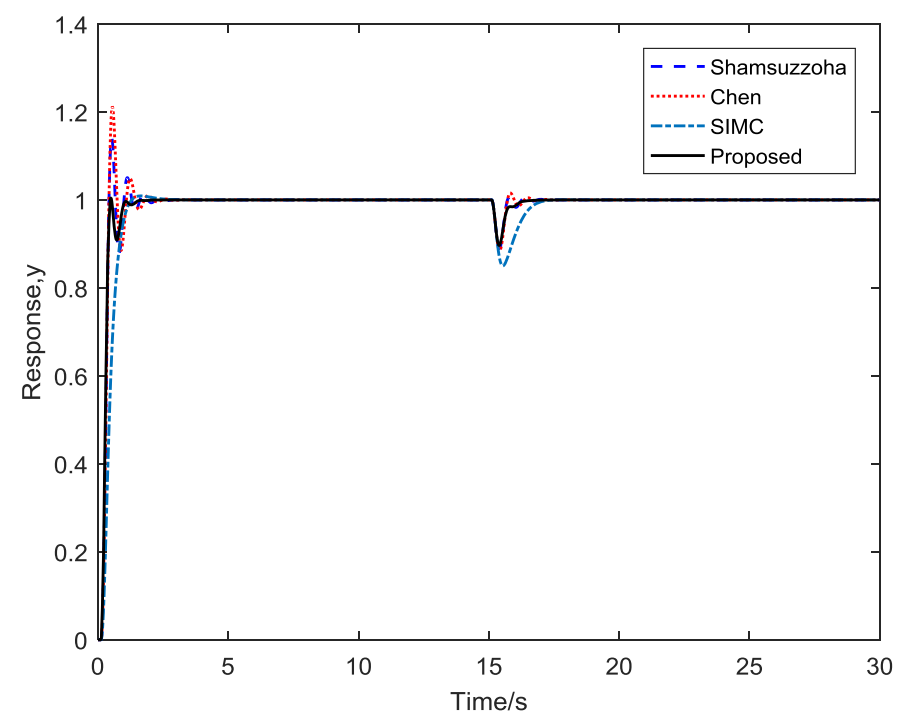

Fig. 6. Response of the yarn tension control system with $10 \%$ parameters.

Table 2. PID controller parameters and the resulting performance indices.

\begin{tabular}{|c|c|c|c|c|c|c|c|c|c|}
\hline \multirow[t]{2}{*}{ Method } & \multirow[t]{2}{*}{$\lambda$} & \multirow[t]{2}{*}{$K_{p}$} & \multirow[t]{2}{*}{$K_{I}$} & \multirow[t]{2}{*}{$K_{D}$} & \multirow[t]{2}{*}{$M s$} & \multicolumn{2}{|c|}{ Perfect model } & \multicolumn{2}{|c|}{$10 \%$ mismatch } \\
\hline & & & & & & IAE & Overshoot & IAE & Overshoot \\
\hline Proposed & 0.2146 & 0.121 & 0.231 & 0.0075 & 2.26 & 0.358 & 1.00 & 0.358 & 1.00 \\
\hline Chen & 0.131 & 0.121 & 0.246 & 0.0086 & 2.26 & 0.380 & 1.14 & 0.431 & 1.21 \\
\hline Shamsuzzoha & 0.094 & 0.128 & 0.261 & 0.01 & 2.26 & 0.359 & 1.06 & 0.405 & 1.14 \\
\hline SIMC & 0.1 & 0.065 & 0.081 & 0.0056 & 1.45 & 0.660 & 1.03 & 0.619 & 1.01 \\
\hline
\end{tabular}

There are many perturbations in the winding process. The lateral vibration of the moving yarn makes the yarn tension fluctuate continuously. The lateral vibration of the yarn also causes considerable problems in achieving high quality textiles. The lateral vibrations are mainly caused by the unpredictability and change in the unwinding roller diameter from a full to empty spool. Therefore, we proposed that the active roller be used to nip the moving yarn and reduce the lateral vibrations of the moving yarn. The nip roller is driven by an AC servo motor to adjust the yarn speed. The nip rollers are made of aluminum alloy [28]. Oil lubrication is applied to reduce friction between nip rollers [29].

Figure 7 shows the active roller in the tension control system. If the yarn tension exceeds the limits, the yarn against the active roller will skid, which may influence the yarn tension. The active roller is not commonly used in practical use systems, so the proposed solution, which utilizes the active roller, is only suitable for microtension control systems. The active roller can couple the linear velocity and angular velocity of the moving yarn, which simplifies the control process. Springs are used to tune the nip force between the active roller and the yarn to avoid skidding. The active roller and winding roller are controlled by $\mathrm{AC}$ servo motors, and the parameters of the AC servo motor are as follows:

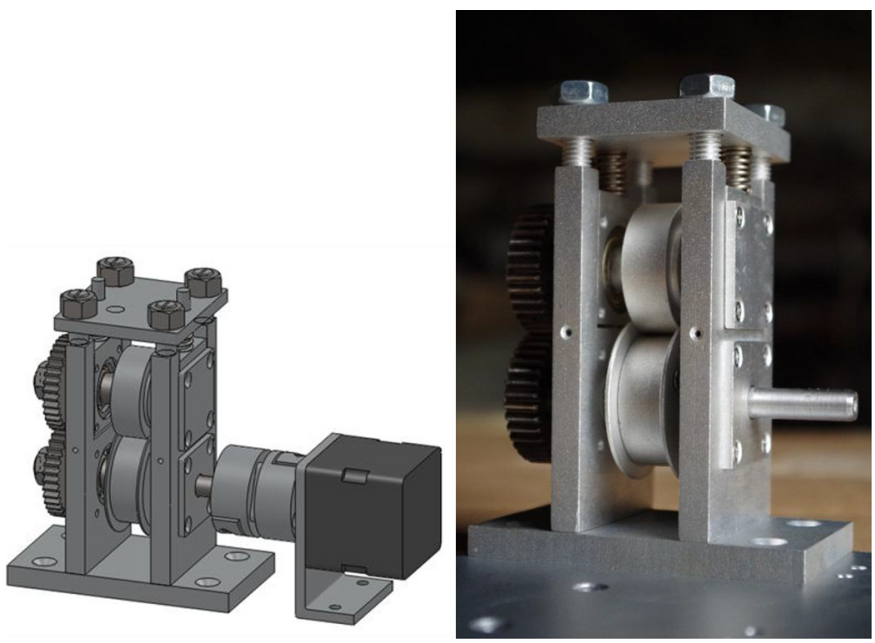

Fig. 7. Active roller of the tension control system.

Rated output power: $50 \mathrm{~W}$; Moment of inertia: $0.025 \times$ $10^{-4} \mathrm{~kg} \times \mathrm{m}^{2}$; Rated torque: $0.16 \mathrm{~N} \times \mathrm{m}$. Rated revolution: $3000 \mathrm{rpm}$.

Figure 8 shows the experimental instrument of the yarn tension control system. As a result of the low precision of 


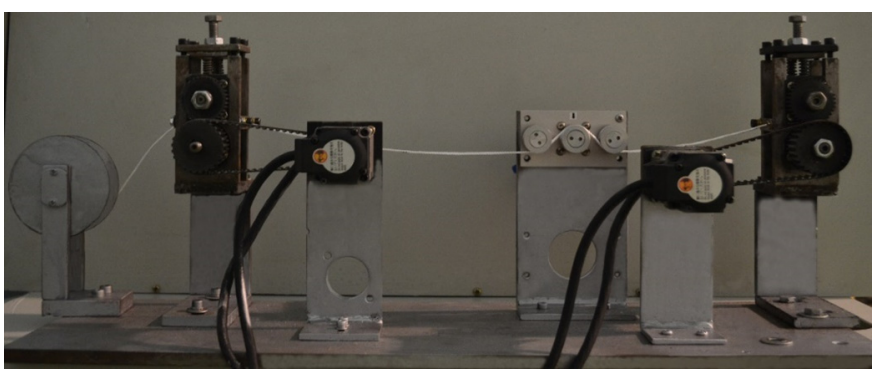

Fig. 8. Experimental instrument of the yarn tension control system.

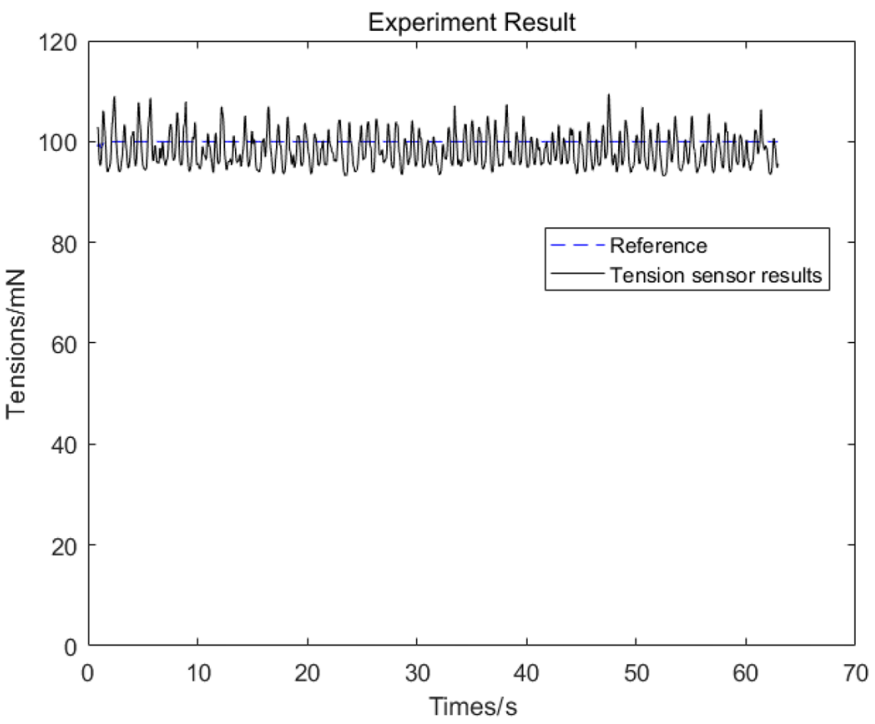

Fig. 9. Yarn tension for reference equal to $100 \mathrm{mN}$.

the instruments and the variation in the diameter of the winding roller, the linear velocity fluctuates periodically with some disturbances. The variation in the yarn tension will affect the quality of the textiles and the winding system directly. The tension therefore must be adjusted by changing the linear velocity of the active roller. We add some disturbances to the linear velocity to simulate the variations in the linear velocity. We proposed feedforward compensation and an IMC PID controller to control the yarn tension of the winding system within a micro range. In textile processes, the yarn input tension can be a major disturbance source, and one of the sources that contributes to disturbances in the input tension is the change in the winding diameter from an empty spool to a full spool. These disturbances are included in the model by changing the linear velocity of the winding roller to simulate the real situation $[30,31]$.

To evaluate the robustness of the proposed method in the real world, we set the reference tension to $100 \mathrm{mN}$. Figure 9 shows the results of the experiment.

The tension static difference ratio and tension fluctuation ratio are two important indicators to evaluate the performance of the tension control system.

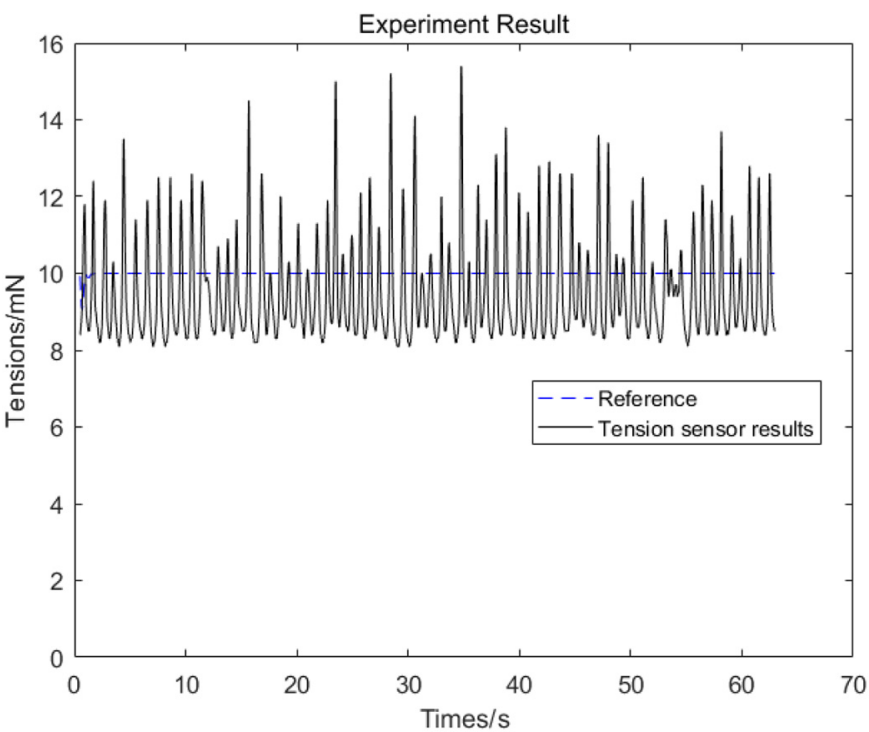

Fig. 10. Yarn tension for reference equal to $10 \mathrm{mN}$.

The tension static difference ratio is calculated as follows:

$$
T_{\delta}=\frac{2\left(T_{\max }-T_{\min }\right)}{T_{\max }+T_{\min }} \times 100 \% .
$$

The tension fluctuation ratio is calculated as follows:

$$
T_{\varepsilon}=\frac{T_{\max }-T_{\min }}{R} \times 100 \% .
$$

From equations (26) and (27), we can calculate $T_{\delta}=15.97 \%$ and $T_{\varepsilon}=16.18 \%$. Because the yarn tension is maintained in a micro-boundary range, the tension static difference ratio and tension fluctuation ratio are larger than typical values of large tension levels, but they were within an acceptable range.

Maryam Naebe tested three different levels of tension and analyzed the effect of yarn winding tension on the Wool Comfort Meter [10]. The results confirmed the significant effect of yarn tension on the Wool ComfortMeter value of the yarn. Because a lack of applied tension will cause the winding yarn to unwind, yarn tension should be maintained within a micro-boundary range. The reference tension is set to $10 \mathrm{mN}$, and the results of this experiment are shown in Figure 10.

In analyzing the results of the experiment, the proposed method can clearly maintain yarn tension within the correct range of microtension. From Figure 9, the value of the yarn tension was maintained within a micro-boundary range. The yarn tension control system design using the IMC PID method is effective at overcoming time delay problems that exist in the winding process. Given the results shown in Figure 10, we know that the IMC PID controller can keep yarn tension at less than $16 \mathrm{mN}$ and maintain the tension in a micro-level. Due to the friction between the yarn and the tension sensor, some problems may exist in measuring yarn tension. The friction caused by 
contact between the yarn and reference pins used for yarn guidance will affects the measured result. For the microtension control system, the influence of friction is substantial and will make the tension static difference ratio and tension fluctuation ratio larger than typical values of large tension levels. However, the ratios were maintained within an acceptable range. These results suggested the effectiveness of the proposed method.

\section{Conclusions}

An IMC PID controller design for a microtension control system is proposed in this paper and demonstrates good performance in a roll-to-roll system. The main purpose of our work is to calculate the value of the coefficient of the PID controller to keep the yarn tension within a microrange. We used feedforward control to adjust the linear velocity of the winding roller and the IMC method to obtain the parameters for the PID controller. The unwinder-winder system can be considered an axially moving string system, and the lateral vibration of the moving string causes yarn tension to fluctuate continuously. We designed the active roller to nip the moving yarn to reduce lateral vibrations of the yarn. The roller can also couple the linear velocity and the angular velocity of the moving yarn. To simulate yarn input tension and the variations of linear velocity in the winding roller, we add disturbances to the linear velocity. We set the reference tension to $100 \mathrm{mN}$ and then $10 \mathrm{mN}$ to test the effectiveness of the proposed method. The results of the simulation and experiment show that the proposed method is useful. Because of the friction and time delay of the tension sensor and fluctuations of the winding roller velocity, yarn tension fluctuated but was maintained within an acceptable range. From the results of the experiment, it is clear that the proposed method is effective in maintain yarn tension within a micro-boundary range.

Acknowledgements. The authors would like to thank the anonymous reviewers and editors for their very constructive comments and suggestions. This work was supported by Shandong Provincial Natural Science Foundation of China (Grant No. ZR2017MEE039).

\section{References}

[1] N.F. Gusarova, Optical monitoring of yarn during its processing, Opt. Zh. 68, 613 (2001) [J. Opt. Technol. 68, 613 (2007)]

[2] A.E. Cherkassky, B.J. Kit, A computer simulation of yarn breakages in the ring spinning process. Part I: Model structure, Investigation strategy, and Experimental design, J. Text. Inst. 88, 47 (1997)

[3] Q. Wang, W. Ge, C. Lu, G. Zhang, H. Shen, B. Jiang, W. Sun, Yarn speed and length measurement using optical method in real time, Opt. Eng. 57, 104103 (2018)

[4] H. Su, X. Zhang, On the mechanical analysis and control for the tension system of the cylindrical filament winding, J. Text. Sci. Technol. 2, 7-15 (2016)
[5] J. Huang, M.Y. Gao et al., The application of PID controller with dead zone for yarn's constant tension control system, 2013 IEEE 8th Conference on Industrial Electronics and Applications (ICIEA), Australia, 19-21 June 2013, IEEEE

[6] Q. Wang, R. Huang, C. Lu, W. Pan, Yarn break detection using an optical method in real time, J. Opt. Technol. 84, 342-346 (2017)

[7] C.X. Wang, Y.Z. Wang, R.Q. Yang, H. Lu, Research on precision tension control system based on neural network, IEEE Trans. Ind. Electr. 51, 381-386 (2003)

[8] N. Sejri, O. Harzallah, S.B. Amar, Influence du pré-mouillage sur les propriétés mécaniques d'un, fil encollé, Mech. Ind. 11, 385-392 (2010)

[9] V.P. Shankam, W. Oxenham, A.M. Seyam, E. Grant, G. Hodge, Wireless yarn tension measurement, and control in direct cabling process, J. Text. Inst. 100, 400-411 (2009)

[10] M. Naebe, B.A. McGregor, D. Tester, Effect of yarn winding tension on the Wool ComfortMeter value when testing yarns. Text. Res. J. 85, 1198-1206 (2015)

[11] M.P. Millman, M. Acar, M.R. Jackson, Computer vision for textured yarn interlace (nip) measurements at high speeds, Mechatronics 11, 1025-1038 (2001)

[12] P. Zhong, Z. Kang, S. Han, R. Hu, J.Y. Pang, X.Y. Zhang, F.X. Huang, Evaluation method for yarn diameter unevenness based on image sequence processing, Text. Res. J. 85, 369-379 (2015)

[13] K.C. Lin, Observer-Based tension feedback control with friction and inertia compensation, IEEE Trans. Cont. Sys. Technol. 11, 109-118 (2003)

[14] D. Knittel, E. Laroche, Tension control for winding systems with two-degrees-of-freedom $\mathrm{H}_{\infty}$ controllers, IEEE Trans. Ind. Appl. 39, 113-120 (2003)

[15] P. Vivek, Novel Method for Dynamic Yarn Tension Measurement and Control in Direct Cabling Process, PhD thesis, North Carolina State University, Raleigh, USA, 2005

[16] V. Gassmann, D. Knittel, P.R. Pagilla, M.A. Bueno, Fixedorder $\mathrm{H}_{\infty}$ tension control in the unwinding section of a web handling system using a pendulum dancer, IEEE Trans. Cont. Syst. Technol. 20, 173-180 (2012)

[17] M. Shamsuzzoha, Closed-loop PI/PID controller tuning for stable and integrating process with time delay, Ind. Eng. Chem. Res. 52, 12973-12992 (2013)

[18] R.J. Mozhdehi, A.S. Ghafari, Optimal PID control of a nanoNewton CMOS-MEMS capacitive force sensor for biomedical applications, Mech. Ind. 15, 139-145 (2014)

[19] C. Anil, R.P. Sree, Tuning of PID controllers for integrating systems using direct synthesis method, ISA Trans. 57, 211-219 (2015)

[20] S. Skogestad, Simple analytic rules for model reduction and PID controller tuning, J. Process Control 13, 291-309 (2003)

[21] M. Shamsuzzoha, S. Skogestad, The set point overshoot method: A simple and fast closed-loop approach for PID Tuning, J. Process Control 20, 1220-1234 (2010)

[22] R.J. Mozhdehi, A.S. Ghafari, Optimal PID control of a nanoNewton CMOS-MEMS capacitive force sensor for biomedical applications, Mech. Ind. 15, 139-145 (2014)

[23] R.P. Nachane, K.R.K. Iyre, Yarn tension as a function of extension: a new approach, Text. Res. J. 57, 279-282 (1987)

[24] Z.Y. Xi, C.H. Lu, J.C. Zhang, Modeling of computercontrolled AC servo feed system, Mater. Sci. Forum 471, $216-220(2004)$ 
[25] Q. Wang, C.H. Lu, W. Pan, IMC PID controller tuning for stable and unstable processes with time delay, Chem. Eng. Res. Des. 105, 120-129 (2016)

[26] Y. Zhang, Q.G. Wang, K.J. Astrom, Dominant pole placement for multi-loop control systems, Automatica 38, $1213-1220$ (2002)

[27] D. Chen, D.E. Seborg, PI/PID controller design based on direct synthesis and disturbance rejection, Ind. Eng. Chem. Res. 41, 4807-4822 (2002)

[28] M. Shamsuzzoha, M. Lee, IMC-PID controller design for improved disturbance rejection of time-delayed processes, Ind. Eng. Chem. Res. 46, 2077-2091 (2007)
[29] P. Zhang, Y. Wang, Effects of shear strain and annealing on the nano-precipitate phase and crystal orientation of 7055 aluminum alloy during cutting process. Vacuum 151, $247-253(2018)$

[30] Y. Zhang, C.H. Lu, J.K. Ma, Research on two methods for improving the axial static and dynamic characteristics of hydrostatic lead screws, Tribol. Int. 109, 152-164 (2017)

[31] Q. Li, B. Jicheng, F. Yinsheng, Z.Y. Zhang, Study of wire tension control system based on closed loop PID control in HS-WEDM, Int. J. Adv. Manuf. Technol. 82, 1089-1097 (2016)

Cite this article as: Q. Wang, A. Li, Y. Li, J. Liu, H. Shen, G. Li, Microtension control for a yarn winding system with an IMC PID controller, Mechanics \& Industry 20, 609 (2019) 